\title{
FACTOR DE REDUCCIÓN $\beta$ POR INCREMENTO DE AMORTIGUAMIENTO VISCOSO, PARA ESTRUCTURAS DESPLANTADAS SOBRE ROCA
}

\author{
Juan P Hidalgo Toxqui (1), Sonia E. Ruiz Gómez ${ }^{(2)}$
}

\begin{abstract}
RESUMEN
Se presenta una expresión matemática sencilla para obtener el factor de reducción $\beta$ por amortiguamiento. Este sirve para reducir las ordenadas espectrales para el diseño sísmico de estructuras con amortiguamiento suplementario. La expresión se propone a partir de dos tipos de análisis probabilistas de sistemas de un grado de libertad desplantadas sobre suelo rocoso. Se considera que los sistemas presentan comportamiento elástico lineal elástico, y alternativamente, comportamiento elastoplástico. En ambos tipos de análisis se toman en cuenta diferentes porcentajes de amortiguamiento crítico (entre 5 y 30\%). La ecuación propuesta se compara con expresiones publicadas en México y con la recomendada en el documento FEMA 450.
\end{abstract}

Palabras Clave: Factor de reducción; amortiguamiento viscoso; espectro de diseño sísmico; suelo rocoso

\begin{abstract}
A simple mathematical expression to obtain the damping factor $\beta$ is presented. It can be used to reduce the spectral ordinates in order to design structures with added viscous damping. Factor $\beta$ is obtained from the analysis of single-degree-of-freedom-systems with elastic-linear and, alternatively, with elastoplastic behavior, using two probabilistic approaches. The systems are located on firm soil. In both types of analyses different percentages of critical damping in the structures are used (between 5 and 30\%). The equation proposed is compared with expressions published in Mexico and with that recommended by FEMA 450.
\end{abstract}

Key Words: Reduction factor, viscous damping; seismic design spectrum; firm ground

\section{INTRODUCCIÓN}

El diseño de edificios sismo-resistentes con elementos disipadores de energía han tomando un gran auge a nivel mundial. La incorporación de los amortiguadores viscosos es una opción viable para que las estructuras reduzcan la respuesta dinámica estructural. La inserción de este tipo de dispositivos en las

Artículo recibido el 16 de diciembre de 2009 y aprobado para su publicación el 26 de octubre de 2010. Se aceptarán comentarios y/o discusiones hasta cinco meses después de su publicación.

(1) Estudiante de posgrado en Ingeniería, Instituto de Ingeniería, Universidad Nacional Autónoma de México, Ciudad Universitaria, 04510, México D.F., ic_toxqui@hotmail.com

(2) Investigadora, Instituto de Ingeniería, Universidad Nacional Autónoma de México, Ciudad Universitaria, 04510, México D.F., tel.: 56233654, sruizg@iingen.unam.mx 
estructuras implica reducir las fuerzas de diseño implícitas en las ordenadas espectrales de diseño sísmico mediante cierto factor de amortiguamiento (llamado aquí factor $\beta$ ).

La expresión para el factor $\beta$ se determina a partir de dos tipos de análisis probabilistas de sistemas de un grado de libertad (S1GDL). El primer tipo de análisis se refiere a la técnica de Monte Carlo. Para ello se genera numéricamente un conjunto de acelerogramas simulados y, a partir de ellos, se determinan sus espectros de pseudoaceleración. El segundo tipo de análisis es el Análisis Probabilístico de Peligro de Demanda Sísmica (APPDS). Con este se obtienen espectros con tasa anual de falla uniforme (ETFU, Rivera 2006). A partir de los dos tipos de espectros antes mencionados se realizan cocientes espectrales, y se propone una ecuación simple para obtener el factor de amortiguamiento $\beta$ (que es función del periodo estructural y del porcentaje de amortiguamiento crítico efectivo).

Los S1GDL que se analizan tienen porcentajes de amortiguamiento crítico iguales a 5, 10, 15, 20, 25 y $30 \%$; ductilidades de diseño iguales a 1 y 2 , y periodos estructurales desde 0.01 hasta 5 s. Los sistemas se suponen desplantados sobre roca.

La ecuación propuesta del factor de amortiguamiento $\beta$ se compara con las publicadas por Arroyo y Terán (2002) y por Pérez Rocha et al. (2007), así como con la recomendada en el documento FEMA 450 (2003).

\section{METODOLOGÍA}

En lo que sigue se explican algunos conceptos básicos y la metodología para realizar los análisis.

\section{Amortiguamiento efectivo del sistema}

El porcentaje de amortiguamiento crítico efectivo $(\zeta$ ) de los sistemas que se analizan se define como la suma del porcentaje que proporciona el sistema básico estructural $\left(\zeta_{S B}\right)$ más el porcentaje de amortiguamiento extra que se introduce a este $\left(\zeta_{\text {ede }}\right)$, es decir:

$\zeta=\zeta_{S B}+\zeta_{\text {ede }}$

En este estudio se supone $\zeta_{S B}=5 \%$.

\section{Factor de amortiguamiento $\beta$}

La ordenada espectral de diseño (representada por el coeficiente sísmico $C_{y}$ ) correspondiente a estructuras con amortiguamiento extra se obtiene multiplicando la ordenada espectral de diseño correspondiente al sistema básico (que tiene un porcentaje de amortiguamiento crítico $\zeta_{S B}$ ) por el factor de reducción por amortiguamiento, $\beta$, es decir:

$$
C_{y}\left(Q_{0}, \zeta, T_{0}\right)=C_{y}\left(Q_{0}, \zeta_{S B}, T_{0}\right) \times \beta\left(Q_{0}, \zeta, T_{0}\right)
$$

donde $C_{y}$ representa el coeficiente de diseño sísmico, $T_{0}$ y $Q_{0}$ son los valores del periodo de vibración y de la ductilidad de diseño de la estructura, respectivamente. 
Por lo tanto, el factor de amortiguamiento $\beta$ resulta igual a (Ruiz y Toxqui, 2008):

$$
\beta\left(Q_{0}, \zeta, T_{0}\right)=\frac{C_{y}\left(Q_{0}, \zeta, T_{0}\right)}{C_{y}\left(Q_{0}, \zeta_{S B}=5 \%, T_{0}\right)}
$$

La ecuación 3 se emplea para obtener los valores de $\beta$ a partir de los espectros obtenidos tanto del análisis de Monte Carlo (espectros de respuesta) como del análisis probabilista de peligro de la demanda sísmica (espectros con tasa anual de excedencia ó de falla uniforme).

\section{Análisis de Monte Carlo}

Los pasos para realizar el análisis de Monte Carlo son los siguientes:

1) Se genera un conjunto de acelerogramas simulados (AS).

2) Se obtienen los espectros de pseudoaceleración, asociados a cada acelerograma y a cada amortiguamiento efectivo del sistema en estudio. Para obtener los espectros de pseudoaceleración se utilizó el programa DEGTRA A4 versión 4.06 (Ordaz y Montoya 2002).

3) Para cada acelerograma se obtienen los factores de reducción, mediante la ecuación 3.

4) Para cada caso de amortiguamiento crítico, se determinan los valores medios de los factores de reducción.

5) Se propone una ecuación y se ajustan sus parámetros.

Los sismos se simularon como procesos estocásticos ergódicos no estacionarios. Estos se obtienen como el producto de un proceso estacionario, $\Psi(t)$, por una función moduladora en el tiempo, $c(t)$ :

$\Phi(t)=c(t) * \Psi(t)$

El acelerograma semilla es el registro obtenido en la estación "Filo de Caballo" ubicada cerca de Chilpancingo, Gro., dirección N00E, el 19 de septiembre de 1985. La estación se encuentra sobre roca, y la distancia focal es de aproximadamente $208 \mathrm{Km}$.

El proceso estacionario $\Psi(t)$ se consideró un ruido blanco filtrado, que se generó como sigue:

$\Psi(t)=\sum_{i=1}^{n} \sigma_{i}\left(a_{i} \operatorname{sen} \omega_{i} \phi_{i}+b_{i} \cos \omega_{i} \phi_{i}\right)$

donde $a_{i}$ y $b_{i}$ representan variables aleatorias independientes con media cero y desviación estándar unitaria, $\phi_{i}$ representa ángulos de fase aleatorios distribuido uniformemente entre 0 y $2 \pi ; \Delta \omega$ indica el intervalo de frecuencias, $n$ el número de intervalos con ancho $\Delta \omega$, y $\sigma_{i}$ representa la desviación estándar:

$\sigma_{i}=\sqrt{2 G_{C P}\left(\omega_{i}\right) \Delta \omega}$

$\boldsymbol{G}_{C P}$ indica la densidad espectral, que aquí se ajusta según el filtro de Clough y Penzien (1975): 
$G_{C P}(\omega)=G_{0}\left[\frac{\omega^{4}}{\left(\omega_{f}^{2}-\omega^{2}\right)+\left(4 \zeta_{f}^{2} \omega_{f}^{2} \omega^{2}\right)}\right]\left[\begin{array}{c}\omega_{g}^{4}+\left(4 \zeta_{g}^{2} \omega_{g}^{2} \omega\right) \\ \left(\omega_{g}^{2}-\omega^{2}\right)+\left(4 \zeta_{g}^{2} \omega_{g}^{2} \omega^{2}\right)\end{array}\right]$

En esta ecuación $\omega_{g} \mathrm{y} \zeta_{g}$ indican el amortiguamiento y la frecuencia equivalente de un "estrato en su primer modo de vibrar"; $\omega_{f}$ y $\zeta_{f}$ representan el amortiguamiento y la frecuencia del "estrato en su segundo modo de vibrar", y $G_{0}$ la magnitud de la densidad espectral del ruido blanco.

En la figura 1a se presenta la función de densidad espectral ajustada. Los valores máximos de la densidad espectral se encuentran entre 7 y $16 \mathrm{rad} / \mathrm{s}$.

Por otro lado, la función moduladora de la intensidad en el tiempo, $c(t)$, del acelerograma semilla, está dada por la ecuación 8 (Yeh y Wen 1989). Las variables independientes $r_{1}, r_{2}, r_{3}, r_{4} y r_{5}$ se ajustaron a la evolución de la Intensidad de Arias en el tiempo.

$c(t)=r_{1} \times \frac{t^{r_{2}}}{r_{4} \times t^{r_{5}}} e^{-t \times r_{3}}$

En la figura $1 \mathrm{~b}$ se muestra la evolución en el tiempo de las amplitudes de aceleraciones cuadráticas, $E\left(a^{2}\right)$, correspondientes al sismo semilla. La fase intensa del sismo se encontró en un intervalo entre 15 y $35 \mathrm{~s}$. La amplitud máxima ocurre a los $25 \mathrm{~s}$, y tiene un valor de $9.4 \mathrm{~cm} / \mathrm{s}^{2}$.

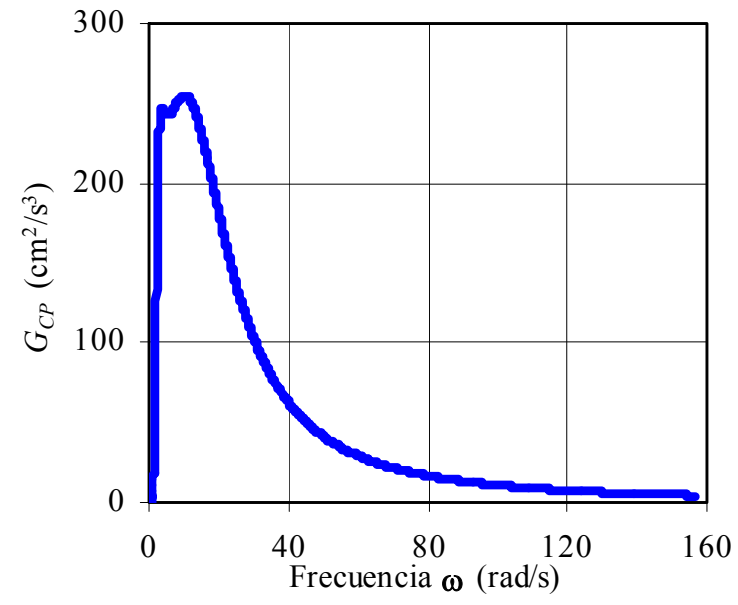

Figura 1a. Función de densidad espectral

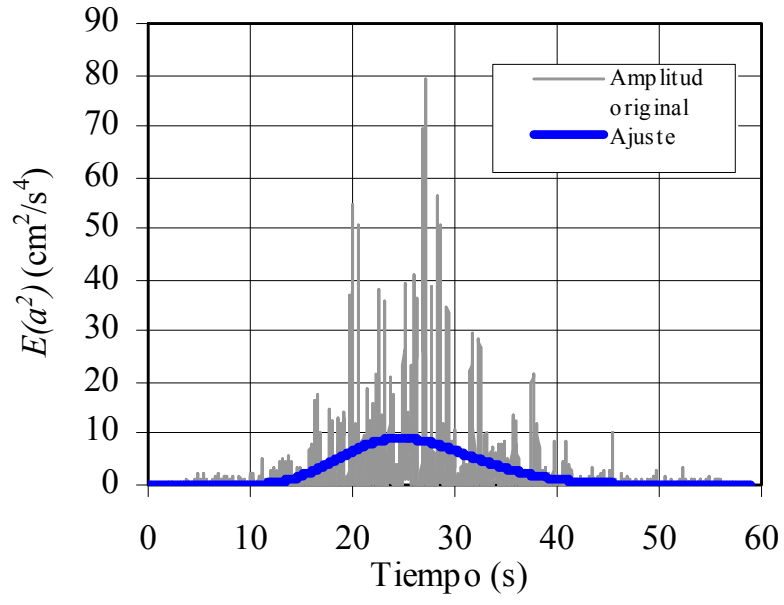

Figura 1b. Función moduladora en el tiempo

\section{Análisis probabilista de peligro de la demanda sísmica}

Mediante un análisis probabilista se determinaron los espectros de peligro uniforme (EPU) y de tasa anual de falla uniforme de las estructuras (ETFU). La metodología para obtener estos últimos fue propuesta por Rivera y Ruiz (2007). Los ETFU se determinaron mediante el programa de cómputo ETFU4 (Rivera 2006), usando el siguiente algoritmo: 
1) Se eligen las propiedades del S1GDL por estudiar. Esto implica fijar valores de: periodo del sistema estructural $\left(T_{e}\right)$, masa $(M)$, ductilidad disponible $(Q)$, coeficiente de amortiguamiento efectivo $(\zeta)$ y valor inicial del coeficiente sísmico $\left(C_{y}\right)$.

2) Se calcula la rigidez lateral, $K=4 \pi^{2} M / T_{e}^{2}$.

3) Se obtiene el desplazamiento de fluencia del sistema $\delta_{\mathrm{y}}=\left(C_{y} M g\right) / K$; donde $g$ es el valor de la gravedad.

4) Se genera numéricamente un conjunto de acelerogramas simulados (AS).

5) Se escalan los sismos simulados de manera que cubran la intensidad de interés $S_{a}$ (Shome y Cornell, 1999). La relación entre el valor de la intensidad de interés y la tasa anual de excedencia se obtiene de las curvas de peligro sísmico del sitio, que se suponen conocidas (PSM, 2004).

6) Se calcula el desplazamiento último $\left(\delta_{u}\right)$ del sistema empleando un método "paso a paso" en el tiempo (aquí se utilizó el método Runge-Kutta de primer orden). La ecuación del movimiento que gobierna el comportamiento de los sistemas histeréticos de los S1GDL es la siguiente (Baber y Wen 1981):

$\ddot{x}+2 \zeta \Omega \dot{x}+\alpha_{2} \Omega^{2} x+\left(1-\alpha_{2}\right) \Omega^{2} z=-a(t)$

donde:

$z=\left\{\alpha_{3} \dot{x}-\rho\left(\left.\alpha_{4} z|\dot{x}| z\right|^{\alpha_{6}-1}+\alpha_{5} \dot{x}|z|^{\alpha_{6}}\right)\right\} / \eta$

En estas ecuaciones $\zeta$ representa la fracción de amortiguamiento efectivo del sistema, $\Omega$ la frecuencia natural de vibrar de la estructura, $z$ la componente histerética (con unidades de desplazamiento), $\alpha_{2}$ la relación entre la rigidez de posfluencia y la rigidez inicial del sistema; $\alpha_{3}, \alpha_{4}$, $\alpha_{5}$, y $\alpha_{6}$ son parámetros del modelo que controlan la amplitud, forma del ciclo histerético y la suavidad de la transición entre los intervalos elástico e inelástico; $\rho$ y $\eta$ son parámetros que controlan el deterioro del sistema. Los parámetros que se emplean en la ecuaciones 9 y 10 dependen del tipo de sistema que se analiza ( $Q=1$ ó $Q=2$, Silva González, 1998).

7) Se calcula la ductilidad demandada $\left(Q_{D E M A N D A D A}\right)$ como el cociente del desplazamiento último entre el desplazamiento de fluencia del sistema. Se aclara que en este estudio no se consideró el daño estructural debido a la repetición de ciclos de histéresis (fatiga por bajo ciclaje).

8) Se simula la ductilidad disponible del sistema.

9) Se determina el valor de $Q_{c}$ que es el cociente de la ductilidad demandada entre la disponible. Se considera que la estructura falla cuando el valor de $Q_{c}$ es igual o mayor que la unidad.

10) Se calcula si la estructura falla, dado un valor propuesto de $\left(C_{y}\right)$.

11) Se obtiene la tasa media anual de falla $\left(v_{C y}\right)$ asociada al coeficiente sísmico $\left(C_{y}\right)$. Para obtener la tasa media anual de falla de las estructuras se utiliza la siguiente integral (Esteva 1968; Cornell, 1969; Esteva y Ruiz 1979):

$v_{C_{y}}\left(C_{y}\right)=\int\left|\frac{d v_{S_{a}}}{d S_{a}}\right| P\left(Q_{c} \geq 1 \mid S_{a}\right) d S_{a}$

donde $v_{C y}\left(C_{y}\right)$ representa la tasa anual de falla de la estructura, $v_{S a}$ la curva de peligro sísmico, $S_{a}$ la intensidad, y $P\left(Q_{c} \geq 1 \mid S_{a}\right)$ la probabilidad que la ductilidad demandada en la estructura sea mayor que su capacidad, dada la intensidad $S_{a}$. 
La probabilidad de falla se calcula aquí como el cociente del número de acelerogramas en los que $Q_{c}$ es mayor que la unidad, entre el número total de movimientos sísmicos a los que se somete la estructura. Aquí se utilizaron 100 acelerogramas simulados (AS). Se eligió este número de movimientos sísmicos debido a que con una cantidad mayor no variaba significativamente la probabilidad de falla.

12) El algoritmo se repite, iterando el valor del $C_{y}$ hasta obtener la tasa anual de falla ( $\left.v\right)$ preestablecida. En este estudio se eligió un valor $v=0.008$, que equivale a un periodo de retorno de 125 años.

13) Una vez obtenido dicho valor de $v$, se varían las propiedades de la estructura (ductilidad, periodo y/o porcentaje de amortiguamiento) y se realiza nuevamente el análisis hasta a obtener el valor de $C_{y}$ asociado a $v=0.008$, para cada caso de estudio.

Se construye el espectro de falla uniforme (ETFU) asociado a la tasa anual de falla seleccionada.

\section{Cálculo de las curvas de peligro sísmico con diferentes amortiguamientos}

Con el fin de obtener los factores de reducción correspondientes a distintos porcentajes de amortiguamiento efectivo $(\zeta=10,15,20,25$ y 30\%) fue necesario generar las correspondientes curvas de peligro sísmico. Estas se construyeron a partir de las curvas de peligro sísmico con porcentaje de amortiguamiento crítico igual a 5\%. Para ello se utilizó la siguiente expresión (Esteva 1976):

$v_{y}(y)=\int_{0}^{\infty} v_{x}\left(\frac{y}{z}\right) f_{\varepsilon}(z) d z=E_{\varepsilon}\left[v_{x}\left(\frac{y}{\varepsilon}\right)\right]$

donde: $x$ indica la variable que considera las propiedades de la función conocida, $y$ la variable que representa la intensidad espectral con propiedades de la función que se desea determinar, $\varepsilon$ la relación entre la intensidad espectral de la función que se desea determinar y de la variable de integración $(\varepsilon=y / z), E_{\varepsilon}$ la esperanza de $\varepsilon, y f_{\varepsilon}$ es la función de densidad de probabilidad de $\varepsilon$ que aquí se supone de tipo lognormal:

$f_{z}(z)=\frac{1}{\sigma z \sqrt{2 \pi}} e^{-\frac{1}{2 \sigma^{2}}(\log (z)-\mu)^{2}}$

donde $\mu$ representa la mediana de $z$, y $\sigma$ la deviación estándar logarítmica de $z$.

La media y desviación estándar logarítmicas de $\varepsilon$ se calcularon a partir de los cocientes entre las ordenadas espectrales correspondientes a los espectros de acelerogramas simulados para sistemas con porcentaje de amortiguamiento crítico efectivo incrementado $(10,15,20,25$ y 30\%) y las ordenadas espectrales correspondientes a los espectros de movimientos simulados para sistemas con $5 \%$ de amortiguamiento crítico.

Las curvas de peligro sísmico se obtuvieron del sistema de cómputo PSM 2004 (Peligro Sísmico en México) desarrollado por el Instituto de Ingeniería de la UNAM y el CENAPRED. En la figura 2 se muestran las CPS de la estación más cercana a "Filo de Caballo" que es la correspondiente a Chilpancingo, Gro., para diversos sistemas con 5\% de amortiguamiento crítico y distintos periodos estructurales $\left(T_{e}\right)$. 


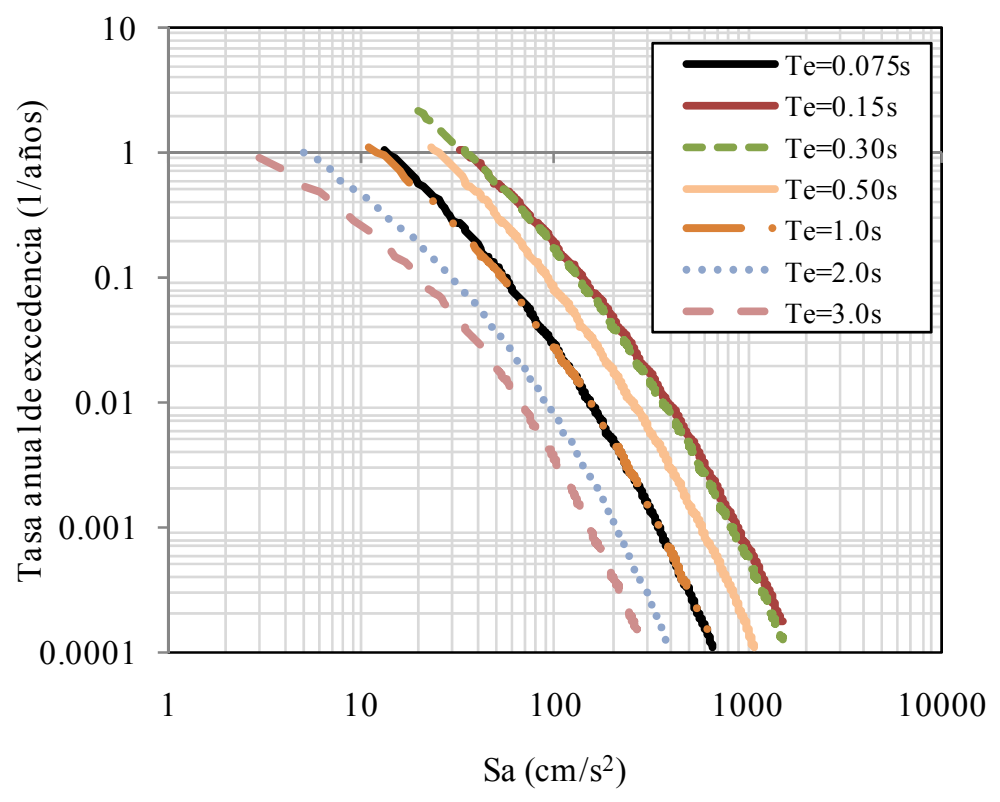

Figura 2. Curvas de peligro sísmico empleadas en este estudio

\section{ESPECTROS SÍSMICOS Y COCIENTES ESPECTRALES}

\section{Espectros de respuesta obtenidos a partir de acelerogramas simulados (AS)}

En las figuras $3 \mathrm{a}$ y b se muestran los espectros de respuesta medios correspondientes a S1GDL con comportamiento lineal $(Q=1)$ y elastoplástico $(Q=2)$, respectivamente, para los seis valores de porcentaje efectivo de amortiguamiento crítico $\zeta=5,10,15,20,25$ y 30\%. Los S1GDL se excitaron con un conjunto de 100 acelerogramas simulados (AS).

En las figuras $3 \mathrm{a} \mathrm{y} \mathrm{b}$ se puede ver la influencia del amortiguamiento viscoso en las estructuras. Como es de esperarse, a medida que aumenta el valor del amortiguamiento, las ordenadas espectrales se reducen. Por otro lado, las ordenadas espectrales correspondientes a sistemas elastoplásticos $(\mathrm{Q}=2)$ son menores que los asociados a los sistemas lineales $(\mathrm{Q}=1)$.

\section{Espectros con tasa anual de falla uniforme (ETFU)}

En la figura 4a se muestran los ETFU para estructuras con $Q=1$. En ellas se puede notar que a medida en que aumenta el porcentaje de amortiguamiento crítico en las estructuras, las ordenadas espectrales se reducen. Lo mismo ocurre para las estructuras con ductilidad $Q=2$. Esto se puede apreciar en la figura $4 \mathrm{~b}$.

\section{Cocientes espectrales}

La figura 5a muestra las medias de los factores de reducción. Estas se calcularon a partir de los cocientes de los espectros correspondientes a acelerogramas simulados (AS) para estructuras con comportamiento lineal $(\mathrm{Q}=1)$. Por otro lado, la figura $5 \mathrm{~b}$ muestra los factores de reducción obtenidos a partir de los cocientes de los ETFU para las mismas estructuras $(\mathrm{Q}=1)$. 


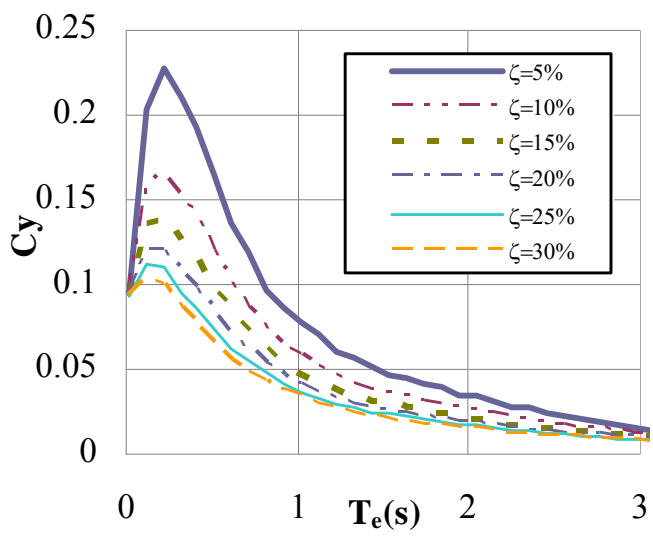

Figura 3a. Medias de los espectros elásticos lineales, usando AS. $Q=1$

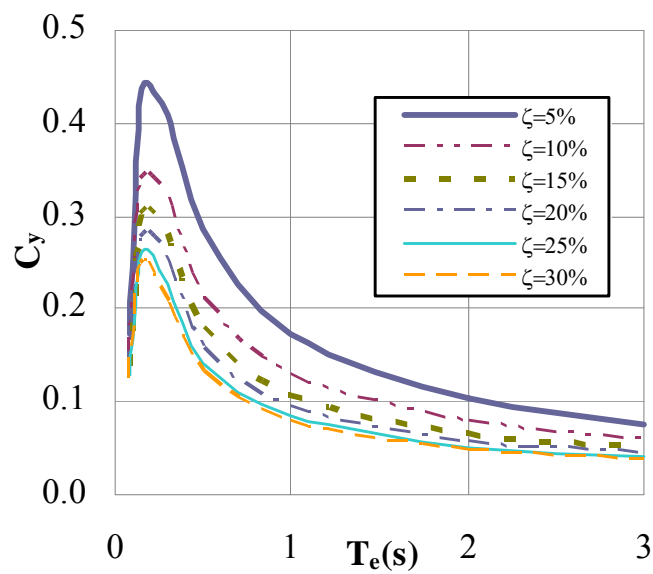

Figura 4a. ETFU para $Q=1$

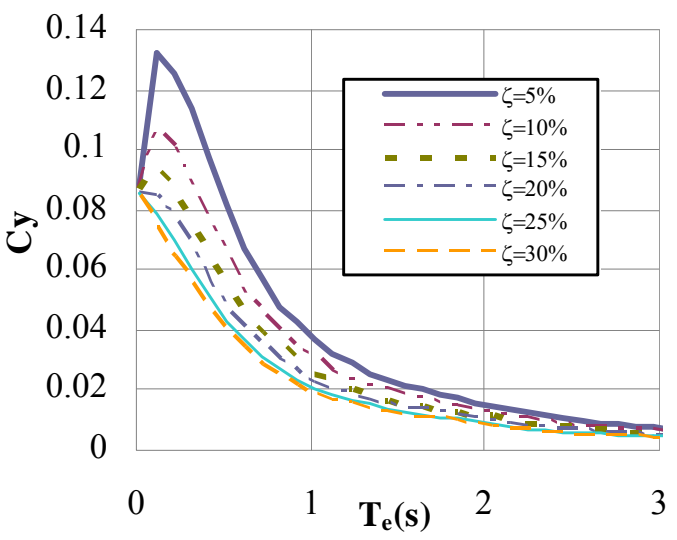

Figura 3b. Medias de los espectros elastoplásticos, usando AS. $Q=2$

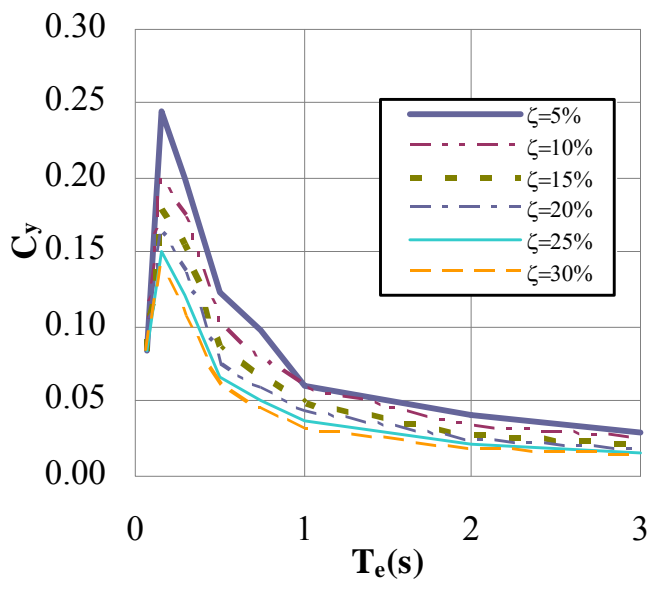

Figura 4b. ETFU para $Q=2$

En las figuras $6 \mathrm{a}$ y b se presentan los factores de reducción $\beta$ para estructuras con comportamiento elastoplástico $(Q=2)$ correspondientes a espectros de acelerogramas simulados (AS) y a ETFU, respectivamente. Los factores tienen un comportamiento similar a los correspondientes a $Q=1$. Se puede observar que los valores de $\beta$ para $Q=2$ (figuras $6 \mathrm{a} \mathrm{y}$ b) son ligeramente mayores que los asociados al caso lineal (figuras $5 \mathrm{a}$ y b); es decir, se observa que los valores de $\beta$ aumentan cuando se incermenta la ductilidad disponible en las estructuras, lo que coincide con los resultados de Wu y Hanson (1989).

\section{FACTOR DE AMORTIGUAMIENTO $\beta$}

Con base en las curvas de las figuras 5 y 6 se propone la siguiente expresión para determinar el factor de reducción por amortiguamiento, $\beta$ : 


$$
\begin{aligned}
& \beta=\left(\frac{\zeta_{S B}}{\zeta}\right)^{\lambda} \\
& \lambda=\left\{\begin{array}{ccc}
\lambda_{0} & \text { si } & \left(T_{e}<T_{c}\right) \\
\lambda_{0}\left(\frac{T_{c}}{T_{e}}\right) & \text { si } & \left(T_{e} \geq T_{c}\right)
\end{array}\right.
\end{aligned}
$$

donde $\zeta_{S B}$ representa la fracción de amortiguamiento crítico de la estructura sin disipadores de energía sísmica, $\zeta$ la fracción de amortiguamiento crítico efectivo en el sistema Estructura-Disipador, y $\lambda_{0}$ es un valor constante que depende del tipo de suelo donde se encuentra desplantada la estructura (Toxqui 2008; Cordero 2010). El periodo $T_{C}$ se adopta igual a $3 \mathrm{~s}$ por tratarse de terreno rocoso (Pérez-Rocha et al, 2007).

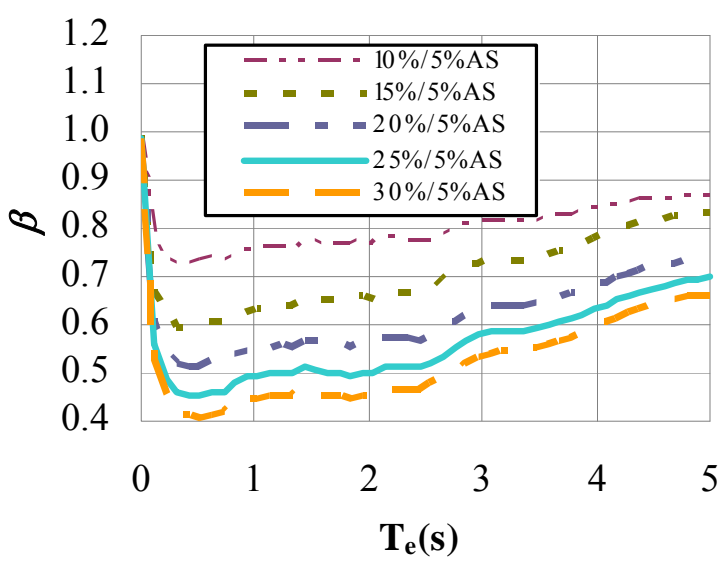

Figura 5a. Factores de reducción usando AS.

$$
\mathrm{Q}=1
$$

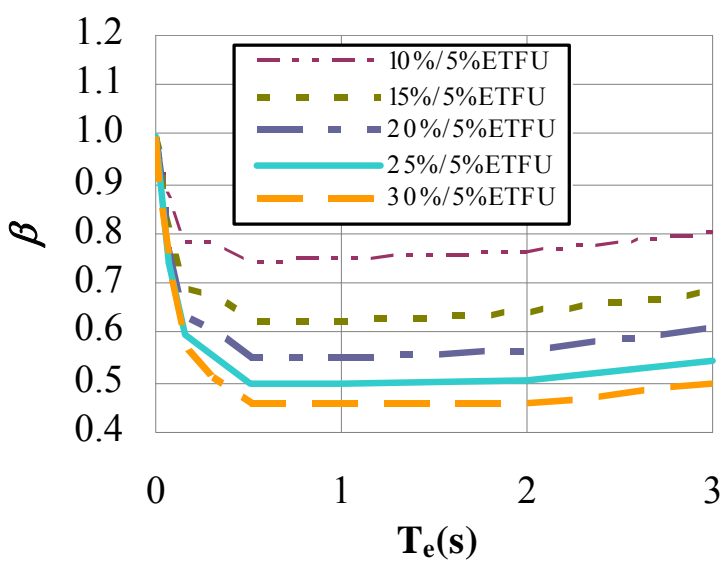

Figura 5b. Factores de reducción usando ETFU. Q=1

\section{Factor de reducción $\beta$ para estructuras con comportamiento elástico lineal $(Q=1)$}

En las figuras $7 \mathrm{a}$ y b se muestran las envolventes propuestas para los factores de reducción $\beta$. Nótese que en dichas figuras no se toma en cuenta que en la parte inicial de cada curva (correspondiente a periodos pequeños) existe una rama descendente de $\beta$, sino que se propone un valor constante desde el periodo estructural $T_{e}=0$ hasta $T_{e}=T_{C}=3 \mathrm{~s}$. El valor de $\lambda_{0}$ que se empleó para ajustar las envolventes en las figuras 7 a y 7 b (líneas continuas) es igual a 0.45 .

En la figura 7a se aprecia un incremento de los valores de $\beta$ para periodos mayores que $T_{C}=3 \mathrm{~s}$. En la figura $7 \mathrm{~b}$ aparecen, con línea continua, las envolventes correspondientes a los factores de reducción encontrados a partir de los cocientes de ETFU. Estas funciones resultan ligeramente sobreestimadas para relaciones de fracciones de amortiguamiento bajo, es decir para $10 \% / 5 \%$ y $15 \% / 5 \%$, pero adecuadas para cocientes altos (por ejemplo, $30 \% / 5 \%$ ). 


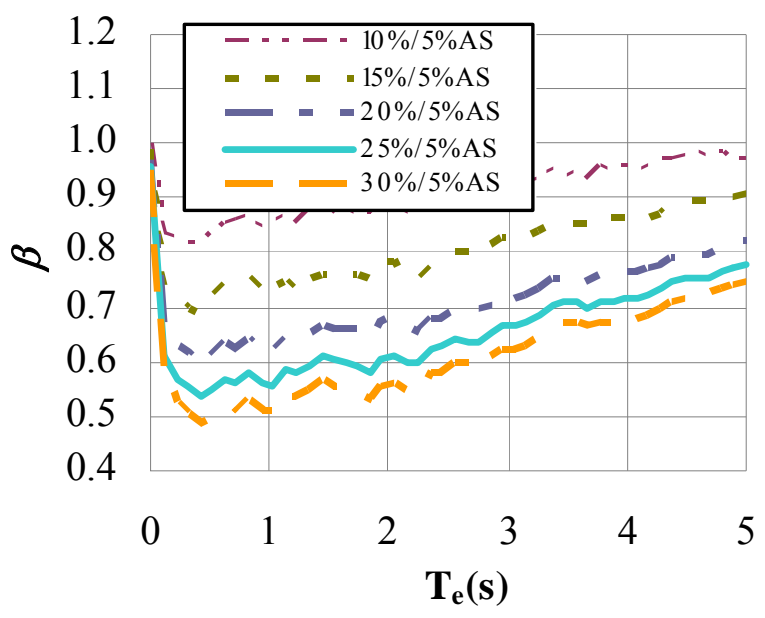

Figura 6a. Factores de reducción usando AS. $Q=2$

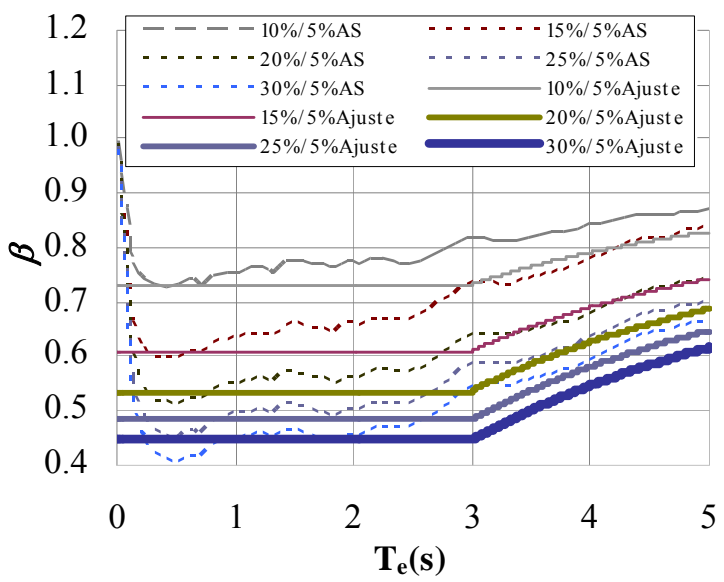

Figura 7a. Factor $\beta$ ajustado usando AS. $Q=1, \lambda_{0}=0.45$

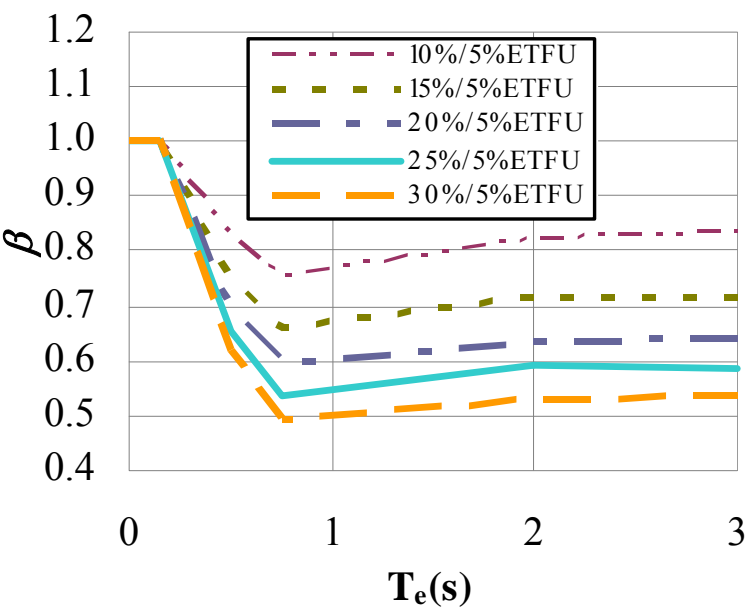

Figura 6b. Factores de reducción usando ETFU. $Q=2$

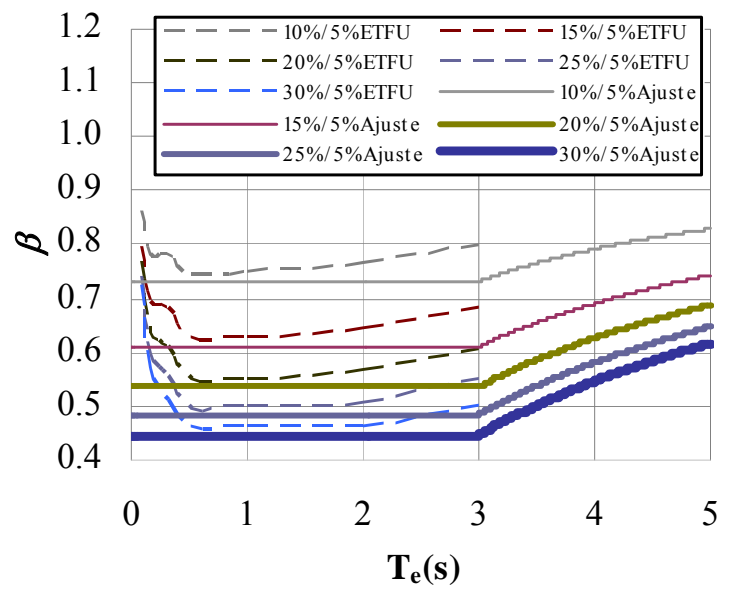

Figura 7b. Factor $\beta$ ajustado usando ETFU. $Q=1, \lambda_{0}=0.45$

\section{Factor de reducción $\beta$ para estructuras con comportamiento elastoplástico $(Q=2)$}

En las figuras $8 \mathrm{a}$ y b se muestran las envolventes para sistemas con $Q=2$. La figura 8 a corresponde a los factores de reducción obtenidos a partir de espectros de respuesta de acelerogramas simulados (AS). Al igual que para el caso lineal, las envolventes presentan valores más cercanos a los valores medios para cocientes de amortiguamiento altos (por ejemplo, 30\%/5\%) que para cocientes bajos (por ejemplo, $10 \% / 5 \%$ ). La figura $8 \mathrm{~b}$ corresponde a envolventes de cocientes de ETFU para $Q=2$. 


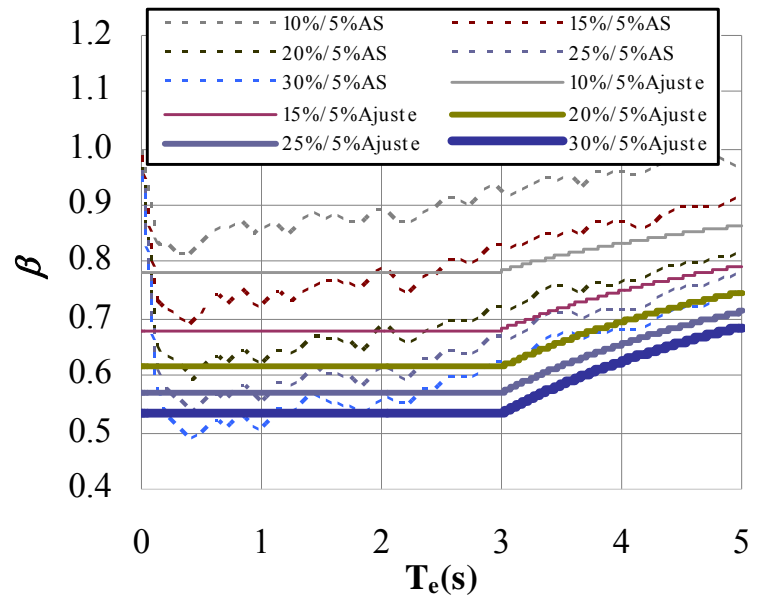

Figura 8a. Factor $\beta$ ajustado usando AS. $Q=2, \lambda_{0}=0.35$

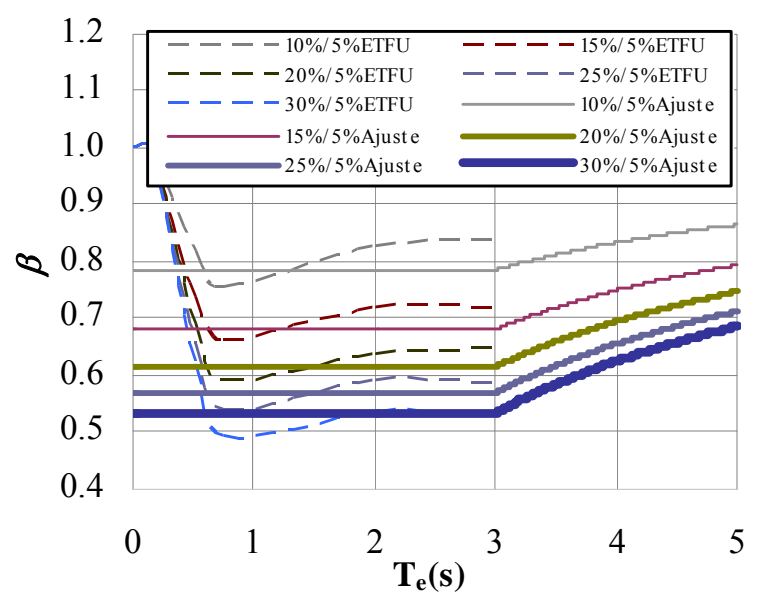

Figura 8 b. Factor $\beta$ ajustado usando ETFU. $Q=2, \lambda_{0}=0.35$

\section{CONSIDERACIONES SOBRE LA EXPRESIÓN PROPUESTA (ECUACIÓN 14)}

Los espectros de diseño sísmico que se usan comúnmente en la práctica inician en la región de aceleraciones $(A)$, partiendo de la aceleración del suelo $\left(a_{0}\right)$. Sus ordenadas se incrementan hasta llegar al región de velocidades $(V)$, en esta región las ordenadas espectrales son constantes, y finalmente, en la región de desplazamientos $(D)$, los valores de $C_{y}$ se reducen a medida que el periodo $\left(T_{e}\right)$ se incrementa (ver figura 9).

Región de aceleraciones (A). Cuando las estructuras son infinitamente rígidas la aceleración absoluta tiende a la aceleración del suelo; es decir, que no es significativo un incremento de amortiguamiento en las estructuras. Esto se puede observar en los espectros de las figuras 3 y 4 . En el presente estudio se verificó que los espectros de diseño que se generan usando la función 14 cumplieran con esta condición.

Región de velocidades $(V)$. Es la región en la que el incremento de amortiguamiento tiene su mayor efecto. Se propone que el factor $\beta$ presente un valor constante a lo ancho de toda esta región.

Región de desplazamiento (D). Cuando las estructuras son infinitamente flexibles su desplazamiento tiende al desplazamiento del suelo. En esta región tampoco es significativo el porcentaje de amortiguamiento inherente en la estructura. (Esto también se puede observar el las figuras 3 y 4 ). Esta condición también se estableció al ajustar la forma de la ecuación 14.

\section{Factor de reducción $\beta$ correspondiente a la "región de aceleraciones" del espectro de diseño sísmico}

En la anterior sección se hizo notar que, para periodos estructurales bajos $\left(T_{e} \leq 0.1 \mathrm{~s}\right)$, la ecuación 14 proporciona valores que sobreestiman los resultados teóricos en comparación a los encontrados analíticamente. En lo que sigue se explica porqué se adopta un valor constante de las envolventes en dicha zona de periodos bajos $\left(0 \leq T_{e} \leq 0.1 \mathrm{~s}\right)$. 


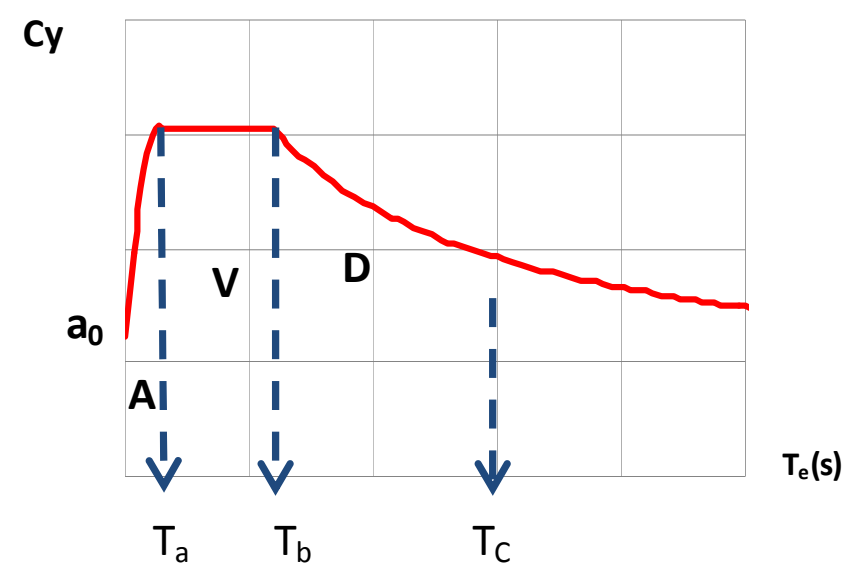

Figura 9. Espectro de diseño sísmico típico

La figura 10a (Caso A) muestra la forma de la expresión que aquí se propone (ecuación 14), mientras que en la figura 10b (Caso B) se presenta una función que podría parecer más apropiada para el factor $\beta$. Este último caso presenta una línea descendente a partir de $T_{e}=0 \mathrm{~s}$ (tal como lo recomienda el FEMA 450, 2003); sin embargo, esta forma (Caso B) da lugar a que se incrementen las ordenadas espectrales de diseño sísmico en la "región de aceleraciones". Esto se ilustra con línea discontinua en la figura 11.

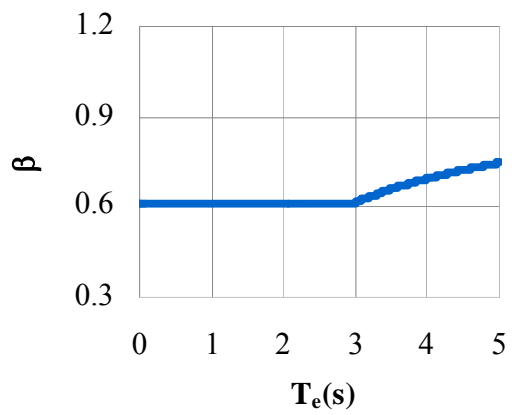

Figura 10a. Factor de reducción para

$$
\zeta_{e}=15 \% \text { y } Q=1 \text {. Caso A }
$$

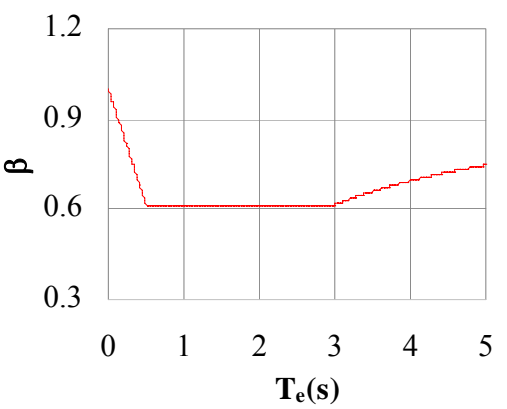

Figura 10b. Factor de reducción para

$$
\zeta_{e}=15 \% \text { y } Q=1 \text {. Caso B }
$$

Con el fin de mantener la forma lineal de la rama ascendente del espectro de diseño, $a=a_{0}+\left(\beta c-a_{0}\right) T / T_{a}$, aquí se propone que la expresión del factor de amortiguamiento (ecuación 14) sea como se representa en la figura 10a (Caso A). 


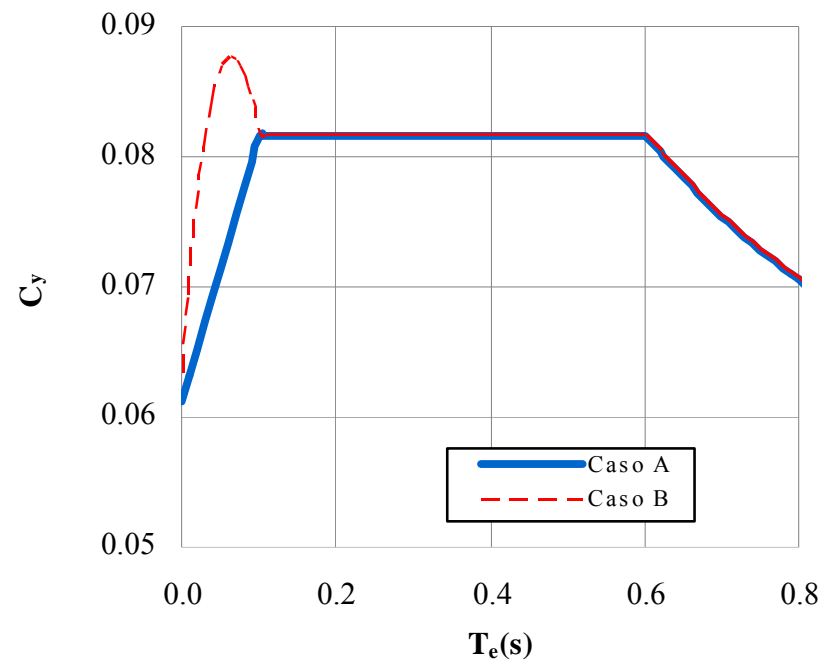

Figura 11. Espectros de diseño sísmico correspondientes a los Casos A y B

\section{Factor de reducción $\beta$ correspondiente a la "región de desplazamiento" del espectro de diseño sísmico}

Por otro lado, la figura 12a (Caso A) muestra el factor $\beta$ con una rama ascendente a partir del periodo $T_{e}=T_{C}$ (ecuación 15), mientras que la figura 12b (Caso C) muestra valores constantes del factor $\beta$ para todos los periodos. Las consecuencias de estas dos alternativas se explican en lo que sigue.

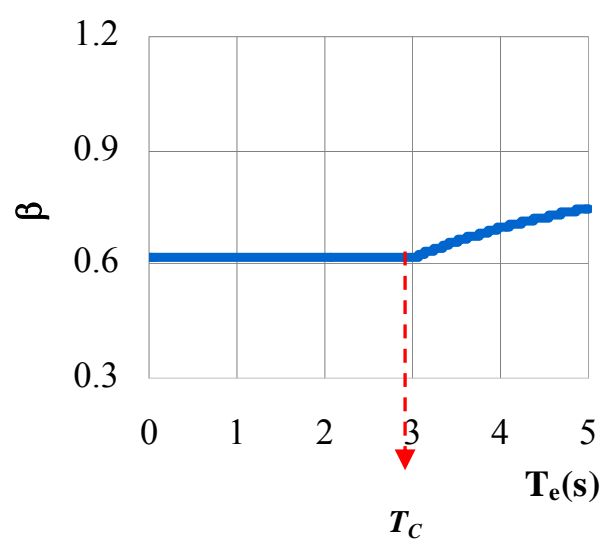

Figura 12a. Factor de reducción para $\zeta_{e}=15 \%$ y $Q=1$. Caso A

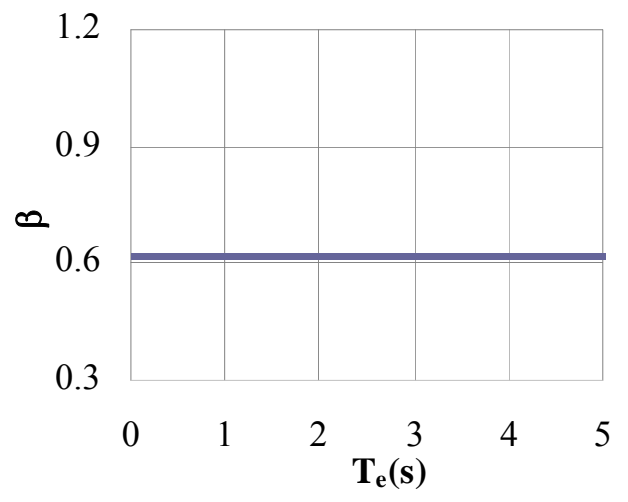

Figura $12 \mathrm{~b}$. Factor de reducción para $\zeta_{e}=15 \%$ y $Q=1$. Caso C

En la figura 13a se puede apreciar que los espectros de diseño sísmico para el Caso A presentan ordenadas espectrales ligeramente mayores (lo que indica están del lado de la seguridad); por el contrario, para el Caso C las ordenadas están del lado no conservador. 
El efecto de utilizar la forma del factor $\beta$ que presenta la figura 12a (en vez de la de la figura 12b) se refleja más claramente en los espectros de desplazamiento. En la figura $13 \mathrm{~b}$ se hace ver que cuando no existe una rama ascendente para $T_{e} \geq T_{C}$ (Caso $\mathrm{C}$ ), los desplazamientos no tienden rápidamente al desplazamiento del suelo, lo que resulta en una estimación inadecuada de dicho desplazamiento; sin embargo, cuando existe una rama ascendente en las envolventes (Caso A), los desplazamientos tienden más rápidamente al desplazamiento del suelo.

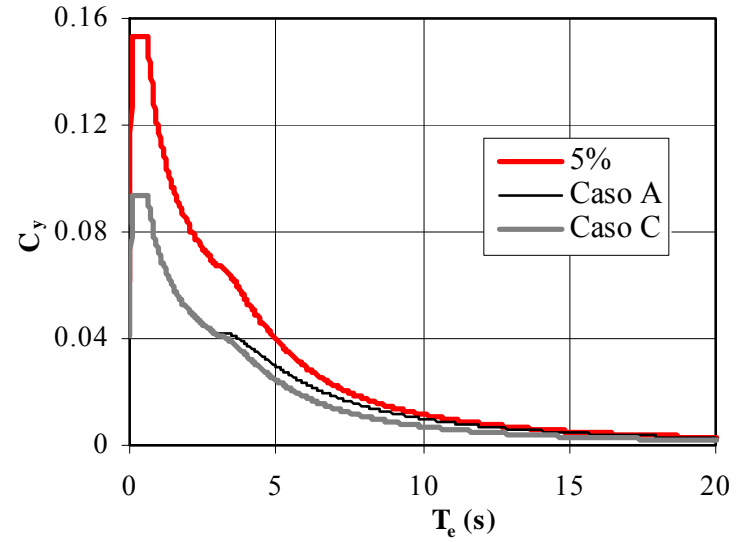

Figura 13a. Espectros de diseño sísmico para el Caso A y el Caso C

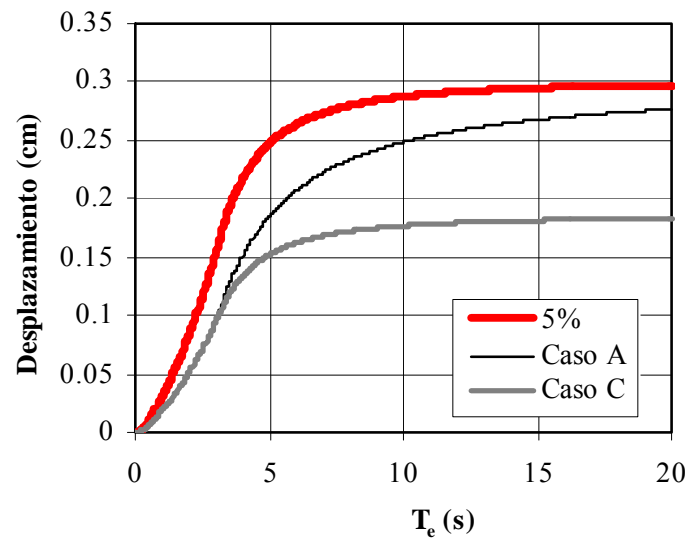

Figura 13b. Espectros de desplazamiento para los Casos A y C

\section{Tasa anual de falla e influencia de la magnitud de los eventos sísmicos en el factor $\beta$}

En el presente estudio se observó que si se emplea una tasa anual de excedencia menor que la seleccionada para este estudio (o sea menor que $v=0.008$ ), los factores de amortiguamiento $\beta$ presentan una variación despreciable; sin embargo, si se emplea una tasa anual de excedencia mayor (por ejemplo, $v=0.1)$ los valores de $\beta$ cambian significativamente.

En la figura 14 se puede ver que los valores de $\beta$ son similares para $v=0.008$ y $v=0.002$; $\sin$ embargo para $v=0.1$, dichos valores resultan mayores para el mismo valor de $\zeta$. La figura 14 corresponde a valores de $\beta$ para una estructura con $Q=1, T_{e}=0.5 \mathrm{~s}$ y diferentes valores de $\zeta$.

Se hace notar que la ecuación 14 y el valor de sus parámetros propuestos en este estudio corresponden a un valor de $v=0.008$ que generalmente se asocia a un estado límite entre seguridad de vidas y cercano al colapso. Es decir, que no son aplicables a otro estado límite (por ejemplo, el de servicio). Para verificar lo anterior se realizó un análisis usando la técnica de Monte Carlo. Se utilizaron registros de intensidad baja, que también se obtuvieron en la estación "Filo de Caballo". Estos fueron causados por eventos de subducción de magnitudes relativamente pequeñas, $\mathrm{M}_{\mathrm{s}}<6.0$. También se utilizaron acelerogramas registrados en la misma estación pero con intensidades altas, causados por eventos de subducción de magnitudes $\mathrm{M}_{\mathrm{s}} \geq 6.5$. Las distancias focales se eligieron del mismo orden (para que no fuese una variable que influyera en los resultados). Los resultados del análisis indicaron que el factor de amortiguamiento depende de la magnitud de los eventos que se utilicen en los análisis (ver Anexo 1), lo que coincide con los resultados de Bommer y Mendis (2005). 


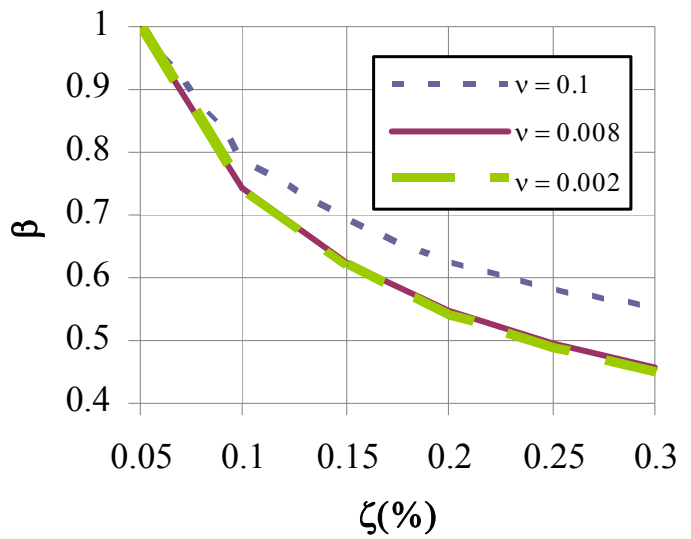

Figura 14. Comportamiento de $\beta$ para distintas tasas anuales de excedencia

\section{COMPARACIÓN DE LA ECUACIÓN 14 CON OTRAS PROPUESTAS}

\section{Comparación del factor $\beta$ (ecuación 14) con las expresión del FEMA 450}

En la figura 15 se compara el factor $\beta$ (con $\zeta=5,10$ y $30 \%$, y $\lambda_{0}=0.35$ ) obtenido con la ecuación 14 con la recomendada en el FEMA 450. La de este último (líneas punteadas) está formada por dos ramas: una recta descendente que llega hasta $T_{0}$ (periodo de referencia donde inicia la meseta en los espectros de diseño sísmico del FEMA 450) y otra recta que es constante para periodos mayores que este valor.

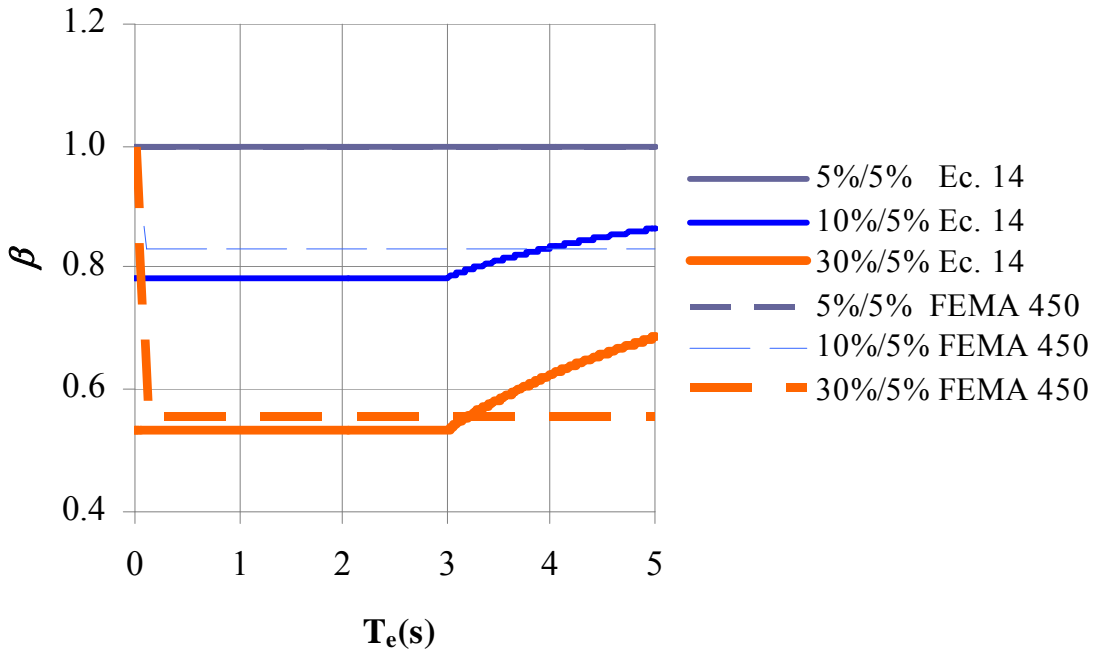

Figura 15. Factores de reducción obtenidos con el FEMA 450 y con la ecuación $14\left(\lambda_{0}=0.35\right)$

Para poder determinar los coeficientes correspondientes al FEMA 450 se estableció la condición que el periodo $T_{0}$ fuese igual al periodo $T_{a}$ (Pérez Rocha et al. 2007), por lo que se supuso que el valor de la constante de aceleración en la respuesta espectral para estructuras en periodos cortos $S_{S}$ fuese igual a 1 (FEMA 450), y el valor de la aceleración en la respuesta espectral para estructuras con periodo estructural 
$\mathrm{T}=1 \mathrm{~s}, S_{1}$ fuese igual a 0.50 . El sistema se supone ubicado en un sitio clase B. Con ello se obtuvo $T_{0}=$ $0.10 \mathrm{~s}$, igual a $T_{a}=0.10 \mathrm{~s}$ (correspondiente a nuestro caso). Los coeficientes del FEMA 450 dividen a las ordenadas espectrales de diseño en vez de multiplicarlas, por lo que en la figura 15 se grafica en valor inverso de lo que el FEMA 450 llama el coeficiente $B$.

En la figura 15 se puede notar que los valores del factor de amortiguamiento son similares en cierto intervalo de periodos; sin embargo, la expresión que recomienda el FEMA 450 daría lugar a que la primera rama del espectro de diseño sísmico no fuese lineal, y por otro lado, posiblemente los desplazamientos no se estimen correctamente (de acuerdo con la explicación de las figuras 11 y 13).

\section{Comparación del factor $\beta$ (ecuación 14) con las expresión de Arroyo y Terán (2002)}

Son pocos los artículos que se encontraron sobre investigaciones relativas a este tema realizadas en México. Uno de ellos es el de Arroyo y Terán (2002), con el que se hace una comparación en lo que sigue. Estos autores proponen la siguiente expresión para encontrar los factores de reducción:

$$
R_{\mu \xi}=\left[\phi\left(\frac{T_{e}^{\kappa}}{\alpha+T_{e}^{\kappa}}\right)+1\right]^{\theta}
$$

donde $\alpha=0.16 \mathrm{y} \mathrm{k}=1.2$

$$
\theta=\frac{0.93-\frac{\kappa-4}{67}}{\frac{1}{T_{e}^{4 \kappa}}}
$$

$\phi=\kappa(3.4 \zeta+1.4)-1.2 \zeta^{-0.1}$ figura 16.

Los factores de reducción dados por la ecuación 16 (para $\zeta=5,10$ y 30\%) se muestran en la

En la figura 16 se puede observar que, cuando no existe amortiguamiento extra en la estructura (5\%/5\%), el valor de $\beta$ que se obtiene con la ecuación de Arroyo y Terán (2002) tiende a un valor ligeramente mayor que la unidad. Esto posiblemente se deba a que la ecuación 16 corresponde a un factor de reducción de resistencia (cociente del espectro elástico entre el inelástico) que depende del periodo, la ductilidad y el amortiguamiento; sin embargo, aquí se emplea para aproximar al factor de reducción por amortiguamiento de una estructura lineal $(\mathrm{Q}=1)$. Solo fue posible comparar sus resultados para el caso de sistemas lineales, debido a la forma en que dichos autores definen el factor $R_{\mu \xi}$.

La figura 16 indica que el factor de reducción obtenido por Arroyo y Terán (2002) es del mismo orden que el calculado con la ecuación 14 cuando se utiliza $\lambda_{0}=0.35$. 


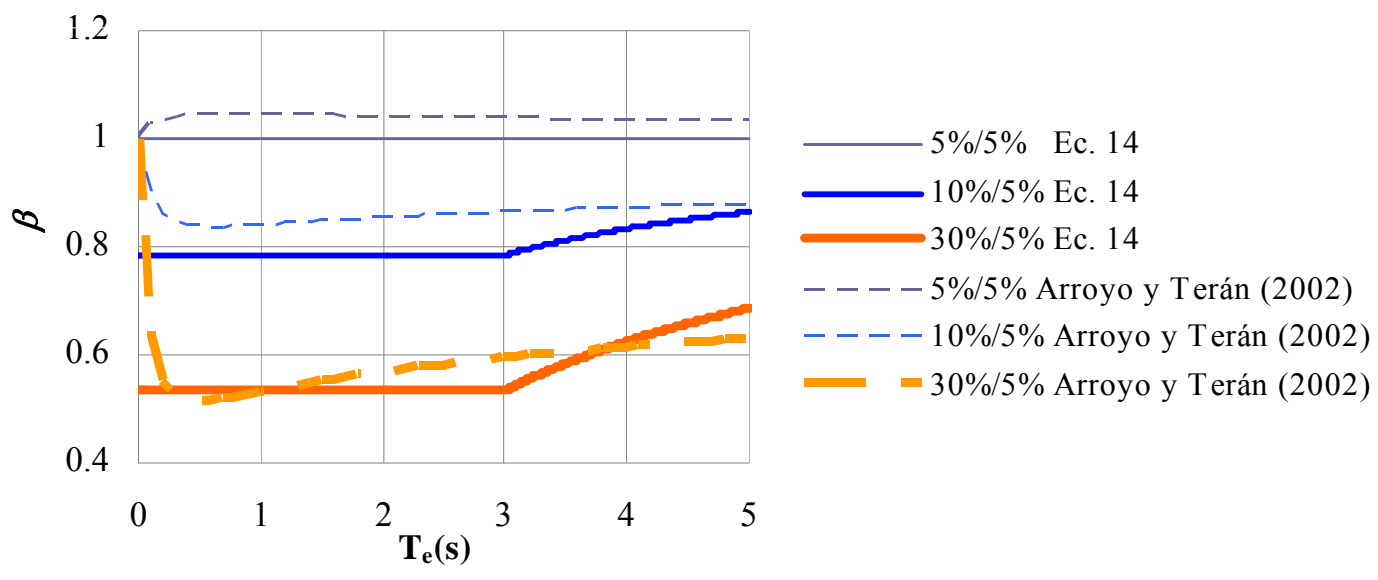

Figura 16. Factores de reducción calculados con la expresión de Arroyo y Terán (2002) y con la ecuación 14. $\lambda_{0}=0.35$

\section{Comparación del factor $\beta$ (ecuación 14) con las expresión propuesta por Pérez Rocha et al (2007)}

La ecuación del factor de amortiguamiento propuesta por Pérez Rocha et al (2007) para obtener el factor de reducción por incremento de amortiguamiento viscoso es la siguiente:

$$
\beta=\left\{\begin{array}{clc}
1+\left[\frac{0.05^{0.45}}{\zeta}-1\right] \frac{T_{e}}{T_{a}} & \text { si } & T_{e} \leq T_{a} \\
\frac{0.05^{0.45}}{\zeta} & \text { si } & T_{a} \leq T_{e}<T_{c} \\
1+\left[\frac{0.05^{0.45}}{\zeta}-1\right] \frac{T_{c}}{T_{e}} & \text { si } & T_{e} \geq T_{c}
\end{array}\right.
$$

donde $T_{e}$ representa el periodo estructural, $T_{a}$ el periodo donde inicia la meseta en el espectro (para suelo rocoso es igual a $0.1 \mathrm{~s}$ ), $T_{c}$ es el periodo donde termina la meseta en el espectro (para suelo rocoso es igual a 3s), y $\zeta$ la fracción amortiguamiento crítico en la estructura con amortiguamiento extra.

En la figura 17 se puede observar que las expresiones de Pérez Rocha et al (2007) contienen una rama descendente del factor de amortiguamiento en el intervalo de periodos pequeños. Esto provocará alteraciones en el espectro de diseño sísmico (como se mencionó al describir las figuras 10 y 11). Es importante notar que los valores $\beta$ propuestos en este estudio son mayores que los obtenidos con las expresiones de Pérez Rocha et al. (2007); sin embargo, cuando se utiliza $\lambda_{0}=0.35$ en la ecuación 14, los valores de $\beta$ se encuentran del lado de la seguridad.

Se hace notar que en la figura 17 los valores de $\beta$ para $20 \% / 5 \%$ obtenidos con las expresiones de Pérez Rocha et al (2007) coinciden con los valores de 30\%/5\% obtenidos con la ecuación 14, por lo que no se notan diferencias entre ambos casos. 


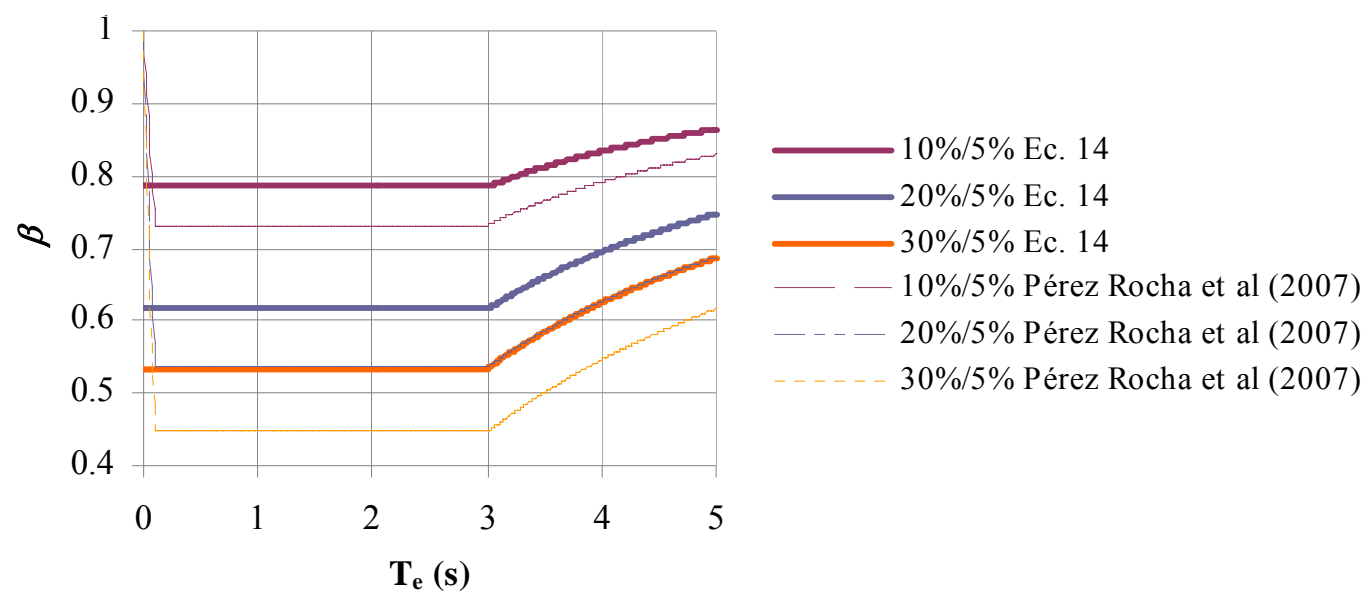

Figura 17. Factores de amortiguamiento obtenidos con la ecuación $14\left(\lambda_{0}=0.35\right)$ y con las expresiones de Pérez Rocha et al. (2007).

\section{CONCLUSIONES}

Se propuso una expresión matemática sencilla para obtener el factor de amortiguamiento $\beta$, útil para reducir las ordenadas espectrales de diseño sísmico para estructuras con amortiguamiento viscoso extra, localizadas en terreno rocoso.

La expresión propuesta depende del porcentaje efectivo amortiguamiento crítico en el sistema $(\zeta)$, del periodo estructural $\left(T_{e}\right)$ y de un parámetro $\left(\lambda_{0}\right)$ que a su vez depende de la ductilidad de diseño $(Q)$. Se encontró que $\lambda_{0}=0.45$ es una valor adecuado para sistemas lineales $(\mathrm{Q}=1), \mathrm{y} \lambda_{0}=0.35$ para sistemas elastoplásticos diseñados con $Q=2$.

La forma de la expresión que se propone es congruente con la de los espectros de diseño sísmico que se utilizan comúnmente para diseño estructural. Los parámetros que se ajustan en este trabajo corresponden a sistemas estructurales sobre roca y sujetos a movimientos sísmicos intensos (que se asocian al estado límite cercano al colapso).

Los resultados que se obtuvieron a partir de registros acelerográficos de baja intensidad sísmica (Anexo 1), junto con la discusión hecha sobre la tasa anual de excedencia referente a la figura 14, indican la necesidad de proponer una nueva forma y/o parámetros para el factor de reducción $\beta$ para sistemas sujetos a movimientos con intensidades pequeñas (generalmente asociadas al estado límite de servicio), así como de ampliar los análisis para estudiar la influencia de la magnitud sísmica y la distancia focal en el factor de reducción amortiguamiento $\beta$. 


\section{AGRADECIMIENTOS}

Se agradecen los valiosos comentarios de J. Avilés López, L. E. Pérez-Rocha y L. Esteva Maraboto a la primera versión de este trabajo. Este estudio se realizó con el apoyo de la DGAPA-UNAM dentro del Programa de Apoyo a Proyectos de Investigación e Innovación Tecnológica (PAPIIT).

\section{REFERENCIAS}

Almora, D, G Castro, R Delgado, L Gaytán, G González-Pomposo, J Herrera, J Iglesias, A Jiménez, M Legorreta, M Macías, S Maldonado, M Moreno, M Navarro, J Pacheco, A Ramírez, M Reséndiz, J A Roldán, R Ruiz, E Rodríguez, H Sandoval, R Silva, E Sordo, M A Soto, A Tello, T Valdéz, C Valdés y V Wong (1995), "Base Mexicana de Datos de Sismos Fuertes", Sociedad Mexicana de Ingeniería Sísmica, AC.

Arroyo, D y A Terán Gilmore (2002), "Factores de reducción de fuerzas sísmicas para el diseño de estructuras con sistemas pasivos de disipación de energía”, Revista de Ingeniería Sísmica, No. 66, pp. 73-93, México.

Baber, T T y Y K Wen (1981), "Random vibration of hysteretic degrading systems", Journal of the Engineering Mechanics Division, 107: EM6, pp. 1069-1087.

Bommer, J y R Mendis (2005), "Scaling of spectral displacement ordinates with damping ratios" Earthquake Engineering \& Structural Dynamics, Vol. 34, pp. 145-165.

Clough, R W y J Penzien (1975), Dynamics of structures, McGraw-Hill, New York

Cordero, S (2010), "Reducción de espectros sísmicos en el sitio viveros debida al incremento del amortiguamiento viscoso en los sistemas estructurales", Tesis de Licenciatura, en proceso, FI UNAM, México.

Cornell, C (1969), “A probability-based structural code", Journal American Concrete, Institute ACI, No.66, pp. 974-985.

Esteva, L (1968), "Bases para la formulación de decisiones de diseño sísmico”, Serie azul 182, Instituto de Ingeniería, UNAM, México.

Esteva, L (1976), "Seismicity, Seismic risk and engineering decisions", Elsevier, pp. 179-224.

Esteva, L y S E Ruiz (1989), "Seismic failure rates of multistory frames", Journal of Structural Engineering, Vol. 115, No. 2, pp. 268-284.

FEMA 450 (2003), “Ground motion”, Chapter 3, Federal Emergency Management Agency, pp. 263-280, Department of Homeland Security, USA.

Grigoriu, M, S E Ruiz y E Rosenblueth (1988), "Nonstationary models of seismic ground acceleration", Earthquake Spectra, Vol. 4, No. 3, pp. 551-568.

Ordaz, M y C Montoya (2002), "Programa DEGTRA A4, Versión 4.06”, Programa de cómputo, Instituto de Ingeniería, UNAM, México.

Pérez-Rocha, L E, J Avilés y E Mena (2007), "Recomendaciones para tomar en cuenta los efectos de sitio en la construcción de espectros de diseño sísmico", XVI Congreso Nacional de Ingeniería Sísmica, Ixtapa-Zihuatanejo, Guerrero, México.

PSM (2004), "Peligro Sísmico en México", Programa de cómputo, Instituto de Ingeniería de la UNAM y el CENAPRED. 
Rivera, J L (2006) "Espectros de confiabilidad uniforme para sistemas estructurales con disipadores de energía", Tesis de Doctorado, DEPFI, UNAM, México.

Rivera, J L y S E Ruiz (2007), "Design approach based on UAFR spectra for structures with displacement-dependent dissipating elements", Earthquake Spectra, Vol. 23, No. 2, pp. 417-439.

Ruiz, S E y J P H Toxqui (2008), "Recomendación para reducir las ordenadas espectrales de diseño sísmico debido a un incremento de amortiguamiento viscoso en estructuras desplantadas sobre suelo duro, factor de amortiguamiento $\beta$ ”, XVI Congreso Nacional de Ingeniería Estructural, Veracruz, Ver.

Shome, N y C A Cornell (1999), "Probabilistic seismic demand analysis of nonlineal structures", Report No. RMS-35, Department of Civil Engineering, Universidad de Stanford, USA.

Silva González, F L (1998), "Calibración del método de linealización equivalente estocástica para sistemas histeréticos simples”, Tesis de Maestría, UNAM, México.

Toxqui, J P (2009), "Reducción de las ordenadas espectrales debida al incremento de amortiguamiento viscoso", Tesis de Maestría, UNAM, México.

Wu, J y R D Hanson (1989), "Study of inelastic respond spectra with high damping", Journal of Structural Engineering, Vol. 115, No.6, pp. 1412-1431.

Yeh, C H y Y K Wen (1989), "Modeling nonstationary earthquake ground motion and biaxial and torsional response of inelastic structures", Structural Research Series, No. 546, University of Illinois, Urbana, IL

\section{ANEXO 1 \\ FACTOR DE AMORTIGUAMIENTO PARA ACELEROGRAMAS CAUSADOS POR EVENTOS DE MAGNITUD PEQUEÑA}

Enseguida se comprueba la dependencia del factor de amortiguamiento $\beta$ con respecto a la magnitud de los sismos. En este anexo se emplean acelerogramas registrados en campo. Los pasos para realizar el análisis son los siguientes:

1) Se extraen los acelerogramas de la estación "Filo de Caballo", de la Base Mexicana de Datos de Sismos Fuertes (Vol. 2).

2) Se descartan los acelerogramas que se encontraron mal registrados o incompletos.

3) Se separan los movimientos en las direcciones N90E y N00E.

4) Cada uno de los acelerogramas se filtra para eliminar frecuencias bajas, y corregir su línea base.

5) Se obtienen espectros de pseudoaceleración para S1GL elástico lineales $(\mathrm{Q}=1)$.

6) Se determinan los cocientes espectrales (dados por le ecuación 3) para cada acelerograma.

7) Se determina la media de los valores de $\beta$.

Los acelerogramas que se usaron en este Anexo se listan en la tabla A1. A partir de este conjunto de registros se formaron dos grupos. El primer grupo está constituido por eventos con magnitudes $\left(\mathrm{M}_{\mathrm{s}}\right)$ menores que 6, movimientos que dieron lugar a valores máximos de coeficientes sísmicos espectrales menores que 0.05. La mayoría de los acelerogramas que conforman al grupo 1 corresponden a eventos con magnitudes promedio de 4.6, sismos que ocurren frecuentemente en el país y que, en general, no implican un peligro de colapso para las estructuras. 
El segundo grupo está constituido por acelerogramas correspondientes a sismos con magnitudes mayores que 6.5 y que dieron lugar a valores máximos espectrales de $C_{y}$ mayores que 0.05 . El segundo grupo está formado por solo tres eventos sísmicos (eventos 8, 47 y 48 de la tabla A1). Estos son relevantes para la seguridad estructural de los edificios; sin embargo, obviamente son muy pocos para realizar con ellos un análisis estadístico.

Los valores medios de cocientes espectrales $(\beta)$ que se calcularon a partir del primer grupo se muestran en la figura A1. En ellos se puede observar que, partiendo desde 1, alcanzan su valor mínimo cerca de $T_{e}=0.5 \mathrm{~s}$. Para periodos mayores que este, el valor de los cocientes aumenta con una pendiente aproximadamente constante; sin embargo en la figura A2, que corresponde a sismos con mayor intensidad sísmica $\left(\mathrm{M}_{\mathrm{s}}>6.5\right)$, el comportamiento es diferente, ya que a partir de $T_{e}=0.8 \mathrm{~s}$ el valor de los cocientes espectrales crece con una pendiente mucho menos pronunciada (comparar la figura A2 con la 5a).

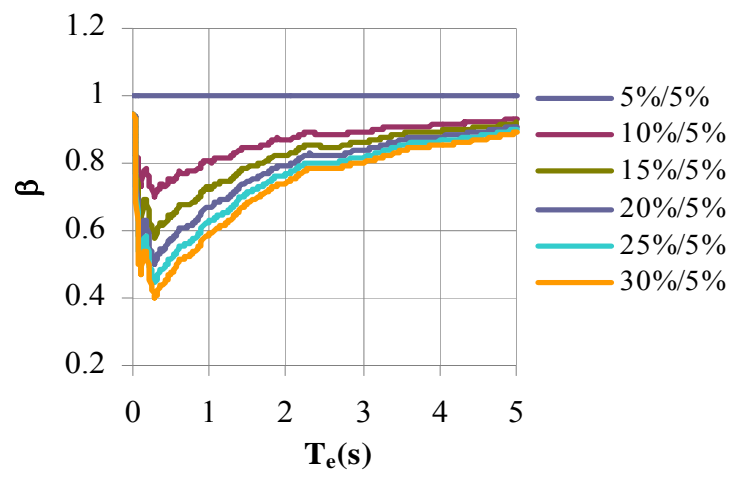

Figura A1. Valores de $\beta$ correspondientes a registros a registros del grupo 1

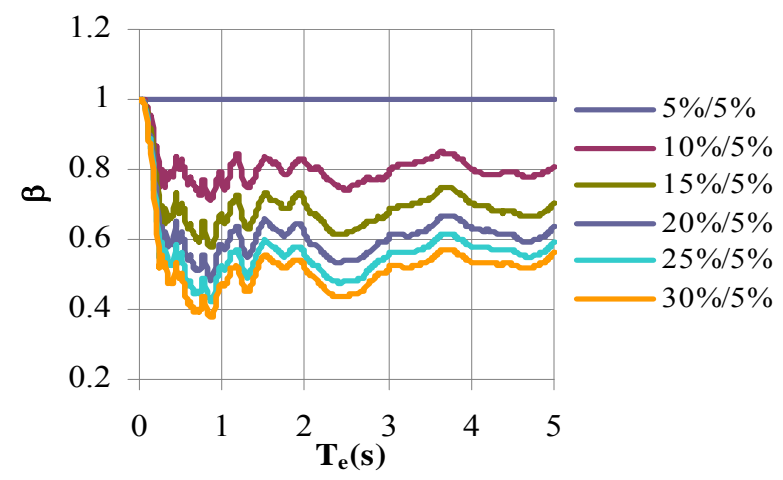

Figura A2. Valores de $\beta$ correspondientes a registros del grupo 2

Al comparar las formas de las curvas que aparecen en las figuras A1 y A2, resulta evidente que los cocientes espectrales, y por lo tanto el factor $\beta$, depende de la magnitud de los eventos sísmicos que se utilice el análisis. 
Tabla A1. Lista de sismos reales empleados en el análisis del Anexo 1

\begin{tabular}{|c|c|c|c|c|c|c|c|c|c|c|}
\hline \multirow{2}{*}{ Número } & \multirow{2}{*}{ Estación } & \multirow{2}{*}{ Archivo } & \multirow{2}{*}{ Fecha del evento } & \multicolumn{3}{|c|}{ Magnitud } & \multicolumn{2}{|c|}{$\begin{array}{c}\text { Coordenadas del } \\
\text { epicentro }\end{array}$} & \multirow{2}{*}{ Duración (s) } & \multirow{2}{*}{ Cy Máximo } \\
\hline & & & & $\mathrm{Mb}$ & Ms & $\mathrm{Mc}$ & $\begin{array}{l}\text { LAT. } \\
\mathrm{N}\end{array}$ & $\begin{array}{l}\text { LONG. } \\
\text { W }\end{array}$ & & \\
\hline 1 & FIC2 & FIC28706091 & 09-jun-87 & 4.2 & & 4 & 16.943 & 99.844 & 21.40 & 0.012 \\
\hline 2 & FIC2 & FIC28701041 & 04 -ene-87 & & & 3.6 & 17.097 & 100.193 & 34.43 & 0.008 \\
\hline 3 & FIC2 & FIC28706071 & 07-jun-87 & 5.5 & 4.8 & 4.9 & 16.654 & 98.909 & 28.86 & 0.022 \\
\hline 4 & FIC2 & FIC28707081 & 08-jul-87 & & & & 16.85 & 99.94 & 34.38 & 0.011 \\
\hline 5 & FIC2 & FIC28805291 & 29-may-88 & 4.6 & & 4.2 & 18.109 & 100.05 & 21.46 & 0.019 \\
\hline 6 & FIC2 & FIC28806161 & 16-jun-88 & & & 4.1 & 18.073 & 99.967 & 42.75 & 0.011 \\
\hline 7 & FIC2 & FIC28808161 & 16-ago-88 & 4.2 & & 4.6 & 16.967 & 99.801 & 21.51 & 0.013 \\
\hline 8 & FIC2 & FIC28904251 & $25-a b r-89$ & 6.3 & 6.9 & 6.5 & 16.603 & 99.4 & 64.90 & 0.058 \\
\hline 9 & FIC2 & FIC28905021 & 02-may-89 & 5.4 & 4.9 & 5.1 & 16.637 & 99.513 & 40.99 & 0.026 \\
\hline 10 & FIC2 & FIC28907071 & 07-jul-89 & & & 4.1 & 17.194 & 99.83 & 20.25 & 0.011 \\
\hline 11 & FIC2 & FIC28908121 & 12-ago-89 & 5.5 & 4.5 & 4.8 & 18.126 & 101.03 & 22.25 & 0.009 \\
\hline 12 & FIC2 & FIC28908171 & 17-ago-89 & 4.9 & & 4.8 & 17.118 & 100.035 & 24.80 & 0.020 \\
\hline 13 & FIC2 & FIC28910081 & 08 -oct- 89 & 5 & 4.1 & 5 & 17.189 & 100.213 & 41.75 & 0.086 \\
\hline 14 & FIC2 & FIC29001131 & 13 -ene-90 & 5.3 & 5 & 5 & 16.82 & 99.629 & 29.97 & 0.034 \\
\hline 15 & FIC2 & FIC29004041 & $04-a b r-90$ & 4.3 & 4.3 & 5 & 16.648 & 99.372 & 23.46 & 0.013 \\
\hline 16 & FIC2 & FIC29005111 & 11-may-90 & & & 4.3 & 17.134 & 100.304 & 20.04 & 0.008 \\
\hline 17 & FIC2 & FIC29005112 & 11-may-90 & 5.3 & 4.9 & 5.3 & 17.046 & 100.84 & 43.98 & 0.020 \\
\hline 18 & FIC2 & FIC29005311 & 31-may-90 & 5.8 & 5.9 & 5.5 & 17.106 & 100.893 & 63.96 & 0.006 \\
\hline 19 & FIC2 & FIC29007101 & 10-jul-90 & 4.5 & & 4.8 & 16.833 & 99.569 & 25.43 & 0.024 \\
\hline 20 & FIC2 & FIC29008201 & 20 -ago-90 & & & 4.3 & 17.167 & 100.027 & 20.73 & 0.013 \\
\hline 21 & FIC2 & FIC29009101 & 10 -sep-90 & 4.6 & & 4.8 & 16.879 & 99.29 & 20.43 & 0.009 \\
\hline 22 & FIC2 & FIC29009171 & 17 -sep-90 & & & 4.3 & 17.04 & 99.822 & 20.61 & 0.010 \\
\hline 23 & FIC2 & FIC29101141 & 14-ene-91 & 5.3 & 5 & 5.1 & 17.838 & 101.854 & 26.61 & 0.011 \\
\hline 24 & FIC2 & FIC29103251 & 25-mar-91 & 4.6 & & 4.7 & 17.165 & 99.867 & 35.32 & 0.072 \\
\hline 25 & FIC2 & FIC29104151 & 15 -abr-91 & 3 & & 4.2 & 17.939 & 99.677 & 21.20 & 0.023 \\
\hline 26 & FIC2 & FIC29104271 & 27 -abr-91 & 4.6 & 4.1 & 4.9 & 17.221 & 100.373 & 35.48 & 0.026 \\
\hline 27 & FIC2 & FIC29105211 & 21-may-91 & 4.9 & 4.1 & 5 & 17.118 & 99.334 & 18.09 & 0.010 \\
\hline 28 & FIC2 & FIC29105281 & 28-may-91 & 4.6 & 3.6 & 4.9 & 16.897 & 99.809 & 24.99 & 0.054 \\
\hline 29 & FIC2 & FIC29107251 & 25-jul-91 & 5.4 & 5.4 & 5 & 16.766 & 101.451 & 21.68 & 0.013 \\
\hline 30 & FIC2 & FIC29108191 & 19-ago-91 & 4.3 & & 4.6 & 17.002 & 100.7 & 23.65 & 0.011 \\
\hline 31 & FIC2 & FIC29110151 & 15 -oct-91 & & & 4 & 17.014 & 99.441 & 21.27 & 0.007 \\
\hline 32 & FIC2 & FIC29203311 & 31-mar-92 & 5.2 & 5.1 & 4.7 & 17.233 & 101.302 & 29.96 & 0.011 \\
\hline 33 & FIC2 & FIC29208021 & 02-ago-92 & 4.3 & & 4.3 & 17.13 & 100.3 & 23.30 & 0.012 \\
\hline 34 & FIC2 & FIC29209191 & 19-sep-92 & & & 4.1 & 17.796 & 100.004 & 22.54 & 0.029 \\
\hline 35 & FIC2 & FIC29210101 & 10 -oct-92 & & & 3.7 & 17.285 & 100.043 & 20.26 & 0.010 \\
\hline 36 & FIC2 & FIC29210161 & 16 -oct-92 & 4.3 & 3.8 & 4.3 & 16.509 & 99.168 & 23.70 & 0.010 \\
\hline 37 & FIC2 & FIC29210251 & 25 -oct-92 & & & 3.8 & 17.151 & 99.965 & 20.58 & 0.012 \\
\hline
\end{tabular}


Facto de reducción $\beta$ por incremento de amortiguamiento viscoso, para estructuras desplantadas ...

\begin{tabular}{rrlrrrrrrrr}
38 & FIC2 & FIC29211101 & 10-nov-92 & 4.6 & 4.2 & 4.3 & 16.887 & 100.101 & 22.03 & 0.015 \\
39 & FIC2 & FIC29212241 & 24-dic-92 & 4.8 & & 4.4 & 16.561 & 99.306 & 21.80 & 0.007 \\
40 & FIC2 & FIC29303311 & 31-mar-93 & 5.3 & 5 & 5.3 & 17.18 & 101.02 & 31.44 & 0.010 \\
41 & FIC2 & FIC29304141 & 14-abr-93 & 3.5 & & 4.6 & 17.04 & 99.46 & 21.09 & 0.010 \\
42 & FIC2 & FIC29307291 & 29-jul-93 & 5 & 4.2 & 5 & 17.38 & 100.65 & 26.49 & 0.019 \\
43 & FIC2 & FIC29308051 & 05-ago-93 & 4.9 & & 5.1 & 17.08 & 98.53 & 22.21 & 0.013 \\
44 & FIC2 & FIC29309101 & 10 -sep-93 & 4.8 & 4.8 & 16.57 & 98.94 & 24.75 & 0.011 \\
45 & FIC2 & FIC29312081 & 08-dic-93 & 4.5 & 4.5 & 17.02 & 99.95 & 20.22 & 0.007 \\
46 & FIC2 & FIC29402231 & 23-feb-94 & 5.4 & & 5 & 17.82 & 97.3 & 22.39 & 0.013 \\
47 & FIC2 & FICA8509191 & 19 -sep-85 & 6.8 & 8.1 & 8.1 & 18.081 & 102.942 & 58.8 & 0.277 \\
48 & FIC2 & FICA8509211 & 21-sep-85 & 6.3 & 7.6 & 7.5 & 18.021 & 101.479 & 33.22 & 0.199 \\
\hline
\end{tabular}

